ESAIM: PROCEEDINGS AND SURVEYS, October 2015, Vol. 51, p. 133-149

A. Garivier et al, Editors

\title{
RANDOM MAPS
}

\author{
Céline Abraham ${ }^{1}$, Jérémie Bettinelli² ${ }^{2}$, Gwendal Collet ${ }^{3}$ and Igor \\ KORTCHEMSKI $^{4}$
}

\begin{abstract}
This is a quick survey on some recent works done in the field of random maps, which, very roughly speaking, are graphs embedded without edge crossings in a surface. We present the main results and tools in this area then summarize the original contributions presented during the conference Journ Âles MAS 2014.
\end{abstract}

\section{INTRODUCTION}

The study of random maps has generated a growing interest in the past decade. Since the pioneering work of Chassaing and Schaeffer [CS04], many contributions came nourishing the field, climaxing with the works of Le Gall [LG13] and Miermont [Mie13], who established the convergence of rescaled uniform plane quadrangulations. This proceeding aims at giving an overview of a few of the most notable results in the field. We refer the interested reader for example to [LGM12] or to Grégory Miermont's Saint-Flour notes for further reading about random maps in general, as well as to the original works presented below for specific details.

Let us first give a proper definition of the objects in question. Unfortunately, although quite natural and easy to comprehend, the concept of map is not very easy to define and the rigorous definition may seem a little bit complicated. We give it nonetheless for self-containment and refer to Figure 1 for an example. A map is a cellular embedding of a finite graph (not oriented and possibly with multiple edges and loops) into a compact connected orientable surface without boundary, considered up to orientation-preserving homeomorphisms. Cellular means that the faces of the map (the connected components of the complement of edges) are open 2-cells, that is, homeomorphic to 2-dimensional open disks. In particular, this implies that the graph is connected. In this paper, all the maps we consider are implicitely rooted, in the sense that they are given with a distinguished oriented edge called the root.

There is also a more visual definition consisting in saying that a map is a gluing of polygons along their boundaries. The most studied maps are by far the maps of the sphere, also called plane maps or planar maps. In this case, the condition of cellular embedding is simply equivalent to the condition of connectedness of the graph. It is also natural to restrict one's attention to particular classes of maps. For example, one might consider triangulations, that is, maps with faces of degree 3 , where the degree of a face is the number of oriented edges encountered when traveling along the boundary of the face. Beware that an edge may be visited twice in this contour, once in one direction, and once in the other one: such an edge will thus be counted twice. For example, on Figure 1, the bottommost face of the map on the left has degree 4. We will see in the next section

${ }^{1}$ Université Paris Sud; celine.abraham@math.u-psud.fr; http://www.math.u-psud.fr/ abraham.

2 CNRS \& Institut Élie Cartan de Lorraine; jeremie.bettinelli@normalesup.org; www.normalesup.org/ bettinel.

3 École polytechnique; gcollet@lix.polytechnique.fr; http://www.lix.polytechnique.fr/Labo/Gwendal.Collet.

4 DMA, École Normale Supérieure; igor.kortchemski@normalesup.org; http://www.normalesup.org/ kortchem.

(c) EDP Sciences, SMAI 2015 

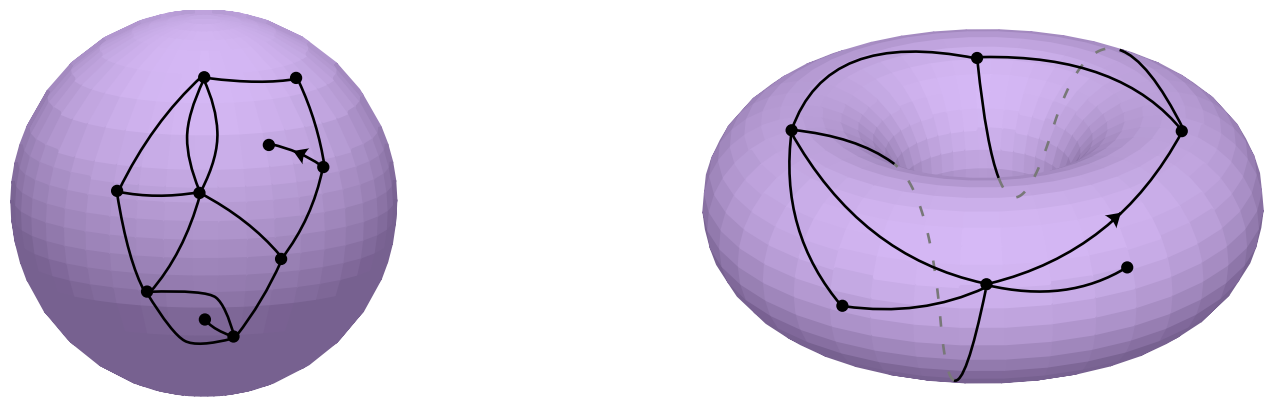

Figure 1. Two maps. The one on the left is a map of the sphere, the one on the right is a map of the torus. The root is the oriented edge represented with an arrow.

that quadrangulations are also central objects (this is due to encoding combinatorial reasons to which we will come back in due course).

Organization of the paper. In the first section, we present a quick overview of the main results concerning limits of random maps. The subsequent sections summarize the original works presented during the random maps session of the Journées MAS 2014.

\section{LIMITS OF RANDOM MAPS}

\subsection{Scaling limits}

Maps come with a natural intrinsic metric given by the graph metric of the underlying graph. Let $\mathfrak{m}$ be a map and let $V(\mathfrak{m})$ denote its vertex-set. The graph metric $d_{\mathfrak{m}}$ is the metric on $V(\mathfrak{m})$ defined by

$$
d_{\mathfrak{m}}(u, v):=\min \{k \geq 0: \text { there exists a chain of } k \text { edges linking } u \text { to } v\}, \quad u, v \in V(\mathfrak{m}) .
$$

Consequently, a map $\mathfrak{m}$ may be seen as a finite metric space $\left(V(\mathfrak{m}), d_{\mathfrak{m}}\right)$ and it is natural to wonder what such a metric space looks like when the map is randomly distributed with a large number of faces. As the metric is growing with the number of faces, one has to rescale the space if one wants to observe a compact limit. This is the point of view of scaling limits.

As we deal with isometry classes of metric spaces, the natural topology is the Gromov-Hausdorff topology introduced by Gromov [Gro99]. The Gromov-Hausdorff distance between two compact metric spaces $(\mathcal{X}, \delta)$ and $\left(\mathcal{X}^{\prime}, \delta^{\prime}\right)$ is defined by

$$
d_{\mathrm{GH}}\left((\mathcal{X}, \delta),\left(\mathcal{X}^{\prime}, \delta^{\prime}\right)\right):=\inf \left\{\delta_{\mathcal{H}}\left(\varphi(\mathcal{X}), \varphi^{\prime}\left(\mathcal{X}^{\prime}\right)\right)\right\}
$$

where the infimum is taken over all isometric embeddings $\varphi: \mathcal{X} \rightarrow \mathcal{X}^{\prime \prime}$ and $\varphi^{\prime}: \mathcal{X}^{\prime} \rightarrow \mathcal{X}^{\prime \prime}$ of $\mathcal{X}$ and $\mathcal{X}^{\prime}$ into the same metric space $\left(\mathcal{X}^{\prime \prime}, \delta^{\prime \prime}\right)$, and $\delta_{\mathcal{H}}$ stands for the usual Hausdorff distance between compact subsets of $\mathcal{X}^{\prime \prime}$. This defines a metric on the set of isometry classes of compact metric spaces, making it a Polish space (see [BBI01]).

The most natural setting is the following. We choose uniformly at random a map of "size" $n$ in some class, rescale the metric by the proper factor, and look at the limit in the sense of the Gromov-Hausdorff topology. The size considered is often the number of faces of the map. From this point of view, the most studied class is the class of plane quadrangulations. The pioneering work of Chassaing and Schaeffer [CS04] revealed that the proper scaling factor in this case is $n^{-1 / 4}$. The problem of scaling limit was then first addressed by Marckert and Mokkadem [MM06], who constructed a candidate limiting space called the Brownian map, and showed the convergence toward it in another sense. Le Gall [LG07] then showed the relative compactness of this sequence of metric spaces and that any of its accumulation points was almost surely of Hausdorff dimension 4. More precisely, he showed the following theorem. 
Theorem 1 (Le Gall [LG07]). Let $\mathfrak{q}_{n}$ be uniformly distributed over the set of plane quadrangulations with $n$ faces. Then, from any increasing sequence of integers, we may extract a subsequence $\left(n_{k}\right)_{k \geq 0}$ such that there exists a random metric space $\left(\mathfrak{q}_{\infty}, d_{\infty}\right)$ satisfying

$$
\left(V\left(\mathfrak{q}_{n_{k}}\right), n_{k}^{-1 / 4} d_{\mathfrak{q}_{n_{k}}}\right) \underset{k \rightarrow \infty}{\stackrel{(d)}{\longrightarrow}}\left(\mathfrak{q}_{\infty}, d_{\infty}\right)
$$

in the sense of the Gromov-Hausdorff topology.

Moreover, regardless of the choice of the sequence of integers, the Hausdorff dimension of the limiting space $\left(\mathfrak{q}_{\infty}, d_{\infty}\right)$ is almost surely 4 .

It is only recently that the solution of the problem was completed independently by Miermont [Mie13] and Le Gall [LG13], who showed that the scaling limit is indeed the Brownian map. This last step, however, is not mandatory in order to identify the topology of the limit: Le Gall and Paulin [LGP08], and later Miermont [Mie08], showed that any possible subsequential limit is homeomorphic to the 2-dimensional sphere. Summing up, we obtain the following theorems.

Theorem 2 (Le Gall [LG13], Miermont [Mie13]). Let $\mathfrak{q}_{n}$ be uniformly distributed over the set of plane quadrangulations with $n$ faces. There exists a random metric space $(M, D)$ called the Brownian map such that

$$
\left(V\left(\mathfrak{q}_{n}\right),\left(\frac{9}{8 n}\right)^{1 / 4} d_{\mathfrak{q}_{n}}\right) \underset{n \rightarrow \infty}{\stackrel{(d)}{\longrightarrow}}(M, D)
$$

in the sense of the Gromov-Hausdorff topology.

Note that the factor $(9 / 8)^{1 / 4}$ does not seem to be of importance in the previous statement but the proper definition of the Brownian map is with this factor. It comes from historical reasons and from an alternative description in terms of random processes, which we will present during Section 1.5.

Theorem 3 (Le Gall [LG07], Le Gall-Paulin [LGP08], Miermont [Mie08]). Almost surely, the Brownian map $(M, D)$ is homeomorphic to the 2-dimensional sphere and has Hausdorff dimension 4.

This line of reasoning lead the way to several extensions. The first kind of extension is to consider other classes of plane maps. Actually, Le Gall already considered in [LG07] the classes of $\kappa$-angulations, for even $\kappa \geq 4$. In [LG13], he considered the classes of $\kappa$-angulations for $\kappa=3$ and for even $\kappa \geq 4$ as well as the case of Boltzmann distributions on bipartite ${ }^{1}$ plane maps, conditioned on their number of vertices. Another extension is due to Addario-Berry and Albenque [ABA13]; in this work, they consider simple triangulations and simple quadrangulations, that is, triangulations and quadrangulations without loops and multiple edges. Quadrangulations without vertices of degree 1 have been considered by Beltran and Le Gall [BLG13]. Bettinelli, Jacob and Miermont [BJM14] later added the case of maps conditioned on their number of edges, and Abraham [Abr13] considered the case of bipartite maps conditioned on their number of edges (see Section 2). In all these cases, the limiting space is always the same Brownian map (up to a multiplicative constant): we say that the Brownian map is universal and we expect it to arise as the scaling limit of many more of natural classes of maps. A peculiar extension is due to Le Gall and Miermont [LGM11] who consider maps with large faces, forcing the limit to fall out of this universality class: they obtain so-called stable maps, which are related to stable processes.

Another kind of extension is to consider quadrangulations on a fixed surface that is no longer the sphere. The case of orientable surfaces with a boundary was the focus of [Bet14], in which the existence of subsequential limits was established. The complete convergence (without the need of extracting subsequences) is the subject of the work [BM14] in preparation:

\footnotetext{
${ }^{1} \mathrm{~A}$ map is called bipartite if its vertex-set may be partitioned into two disjoint subsets such that no edges link two vertices from the same subset.
} 


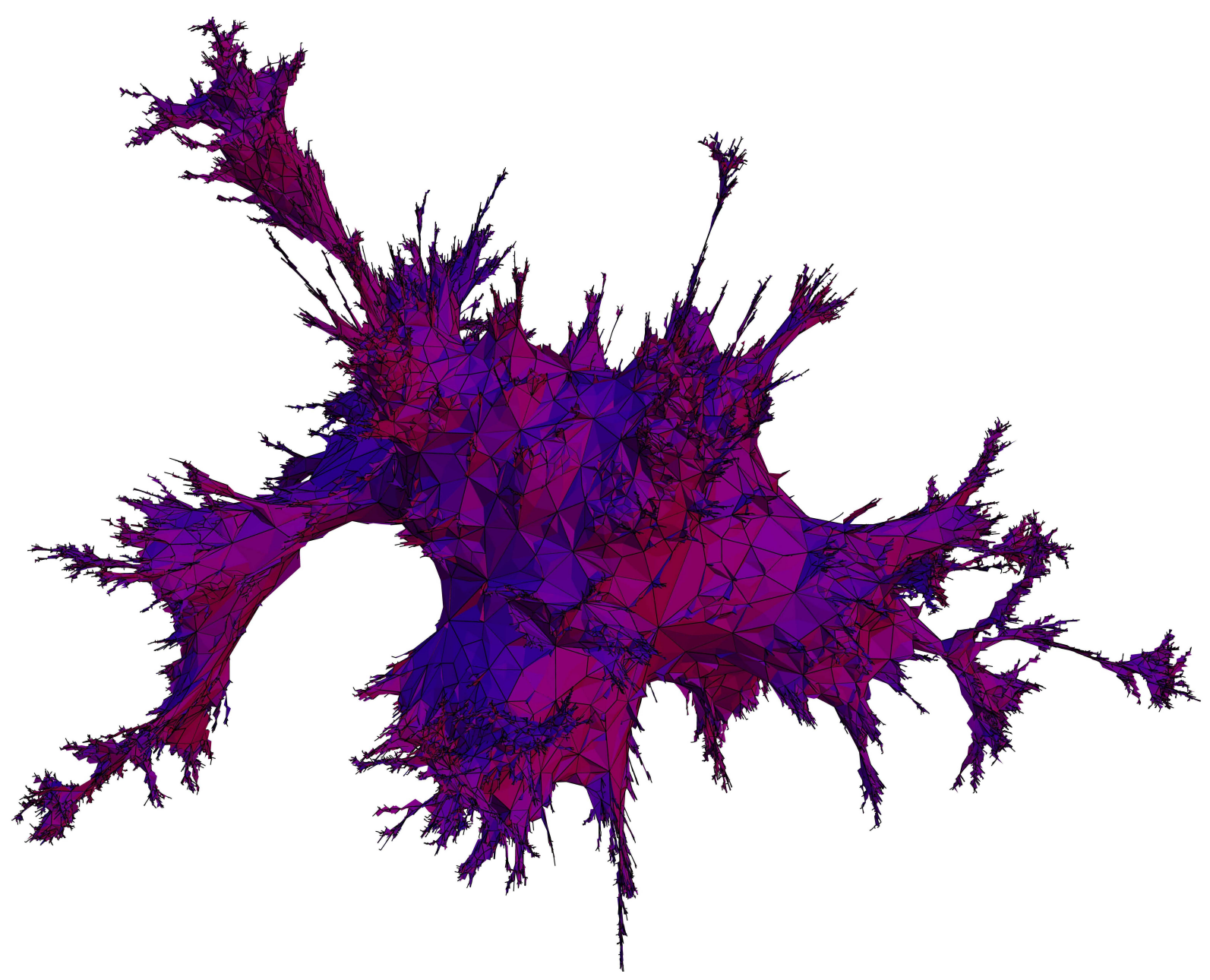

FiguRe 2. Simulation of a uniformly sampled plane quadrangulation with 50000 faces. See http://www.normalesup.org/ bettinel/simul.html for more computer simulations of random maps, in particular of more complicated topology.

Theorem 4 (Bettinelli-Miermont [BM14]). Let $g \geq 0$ be an integer, $\sigma=\left(\sigma^{1}, \ldots, \sigma^{p}\right)$ be a $p$-uple of positive real numbers and $\sigma_{n}=\left(\sigma_{n}^{1}, \ldots, \sigma_{n}^{p}\right) \in \mathbb{N}^{p}$ be such that $\sigma_{n}^{i} / \sqrt{2 n} \rightarrow \sigma^{i}$, for $1 \leq i \leq p$. Let $\mathfrak{q}_{n}$ be uniformly distributed over the set of bipartite genus $g$-maps with $n$ faces of degree 4 and $p$ distinguished faces of respective degrees $\sigma_{n}^{1}, \ldots, \sigma_{n}^{p}$. There exists a random metric space $\left(M_{\sigma}^{(g)}, D\right)$ such that

$$
\left(V\left(\mathfrak{q}_{n}\right),\left(\frac{9}{8 n}\right)^{1 / 4} d_{\mathfrak{q}_{n}}\right) \underset{n \rightarrow \infty}{\stackrel{(d)}{\longrightarrow}}\left(M_{\sigma}^{(g)}, D\right)
$$

where the convergence holds in distribution for the Gromov-Hausdorff topology.

Moreover, almost surely, the limiting space $M_{\sigma}^{(g)}$ is homeomorphic to the surface of genus $g$ with $p$ boundary components, has Hausdorff dimension 4, and every of the p connected components of its boundary has Hausdorff dimension 2. 


\subsection{Geodesics toward a uniformly chosen point}

The metric of the Brownian map $(M, D)$, and a fortiori of more general Brownian surfaces $\left(M_{\sigma}^{(g)}, D\right)$ is quite hard to grasp. Due to their construction, one can however study the geodesics from all the points to a given point uniformly distributed at random. In the Brownian map, Le Gall [LG10] did this study and showed among other properties that, almost surely, the following holds.

- There exists only one geodesic between two uniformly distributed points.

- Given a uniformly distributed point $\rho$, there exist at most 3 distinct geodesics from a point toward $\rho$.

Moreover, he showed the following so-called confluence property of the geodesics.

Proposition 5 (Le Gall [LG10]). Given a uniformly distributed point $\rho$, almost surely, for every $\varepsilon>0$, there exists $\eta \in(0, \varepsilon)$ such that all the geodesics from $\rho$ to points outside of the ball of radius $\varepsilon$ centered at $\rho$ share a common initial part of length $\eta$.

The previous properties were later extended to every Brownian surface $\left(M_{\sigma}^{(g)}, D\right)$ by Bettinelli [Bet14], by using a completely different approach. As, in the general case, the topology is richer than in the case of the sphere, some new interesting characterizations may be made in terms of geodesics. To give only one example, it is shown that, given a uniformly distributed point $\rho$, there is only a finite number of points linked to $\rho$ by three distinct geodesics such that the concatenation of any two such geodesics form a loop that is not homotopic to 0 . This number is random and bounded by an explicit function of the genus and number of boundary components. Its distribution may also be computed. For instance, in the case of a surface of genus $g \geq 1$ without boundary, the situation is simpler: this number is deterministic and equal to $4 g-2$.

\subsection{Encoding by simpler objects: Schaeffer-like bijections}

The starting point of the previous studies is a powerful bijective encoding of the maps in the studied class by simpler objects. In the case of plane quadrangulations, the bijection in question is the so-called CoriVauquelin-Schaeffer bijection [CV81,Sch98, CS04] and the simpler objects are so-called well-labeled trees, that is, trees whose vertices carry integer labels such that the root vertex has label 0 and the variation of labels along the edges are in $\{-1,0,1\}$. In the other cases, variants of this bijection are used [BDG04, CMS09, PS06, AB13] and the encoding objects usually have a more intricate combinatorial structure. In this section, we present the simplest of these bijections, that is, the original Cori-Vauquelin-Schaeffer bijection.

From pointed plane quadrangulations to well-labeled trees. We start from a pointed quadrangulation $\left(\mathfrak{q}, v^{\bullet}\right)$ with $n$ faces, that is, an $n$-faced quadrangulation $\mathfrak{q}$ given with a distinguished vertex $v^{\bullet}$. Remember that $\mathfrak{q}$ is by definition rooted so that $\left(\mathfrak{q}, v^{\bullet}\right)$ really comes with two distinguished elements: the root oriented edge and the distinguished vertex $v^{\bullet}$. First, we assign to every vertex $v$ a label $\hat{\mathfrak{l}}(v):=d_{\mathfrak{q}}\left(v, v^{\bullet}\right)$. Due to combinatorial reasons, the labels of neighboring vertices differ exactly by 1 . As a result, when we travel around a face, the subsequent labels are either $d, d+1, d+2, d+1$, or $d, d+1, d, d+1$, for some integer $d$. We draw inside each face an arc, as depicted on the left part of Figure 3. The embedded graph with vertex-set $V(\mathfrak{q}) \backslash\left\{v^{\bullet}\right\}$ and edge-set the set of newly added arcs is a tree $\mathfrak{t}$, rooted according to some convention which we will not need to discuss in this work. Finally, the vertices of $\mathfrak{t}$ are labeled by translating the function $\hat{\mathfrak{l}}$ in such a way that the root vertex $\rho$ of $\mathfrak{t}$ gets label 0 , that is, we set $\mathfrak{l}:=\hat{\mathfrak{l}}-\hat{\mathfrak{l}}(\rho)$. See Figure 3 .

Note that it is not obvious at first that the object $\mathfrak{t}$ we obtain is a tree as claimed. It is clearly an embedded graph. Then one has to observe that it contains every vertex of $\mathfrak{q}$ except $v^{\bullet}$ and has no cycles. One concludes that it is a union of pairwise disjoint trees (a forest) and the Euler characteristic formula yields that it contains only one tree. All these steps are not very complicated to work out but still demand some time. We refer for instance to [BDG04, Section 2] for proofs.

From well-labeled trees to pointed quadrangulations. Let $(\mathfrak{t}, \mathfrak{l})$ be a well-labeled tree with $n$ edges and let $c_{0}, c_{1}, \ldots, c_{2 n-1}$ denote the sequence of corners of $\mathfrak{t}$, arranged in clockwise order around the tree, starting from the root corner. We extend the list to $\mathbb{Z}_{+} \cup\{\infty\}$ by periodicity, setting $c_{2 k n+i}=c_{i}$ for every $k \in \mathbb{N}$, 

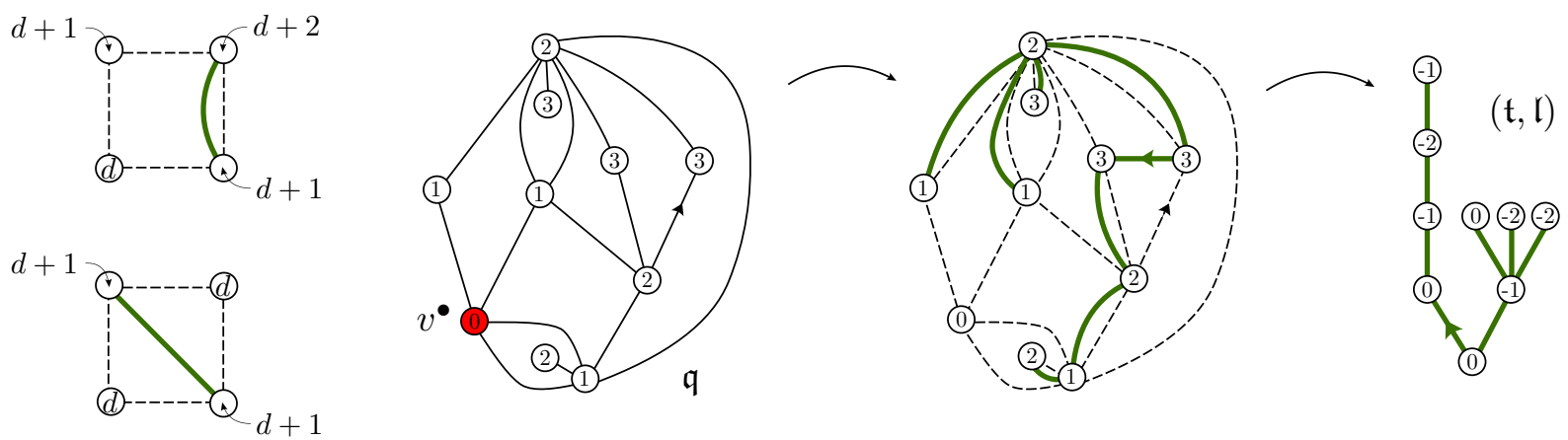

Figure 3. Left. The two types of faces and the extra arc to draw. Right. Construction of the well-labeled tree $(\mathfrak{t}, \mathfrak{l})$ from the pointed quadrangulation $\left(\mathfrak{q}, v^{\bullet}\right)$.

$i \in\{0, \ldots, 2 n-1\}$, and by adding one corner $c_{\infty}$ incident to an extra vertex $v^{\bullet}$ lying inside the unique face of $\mathfrak{t}$. We also assign the label $\mathfrak{l}\left(v^{\bullet}\right):=\min _{V(\mathfrak{t})} \mathfrak{l}-1$. We then define the successor function $s: \mathbb{Z}_{+} \rightarrow \mathbb{Z}_{+} \cup\{\infty\}$ by

$$
s(i):=\inf \left\{j>i: \mathfrak{l}\left(c_{j}\right)=\mathfrak{l}\left(c_{i}\right)-1\right\}, \quad i \in \mathbb{Z}_{+},
$$

where, for a corner $c$, we wrote $\mathfrak{l}(c)$ the label of the incident vertex and, as usual, $\inf \{\varnothing\}:=\infty$. We then set $s\left(c_{i}\right):=c_{s(i)}$. The construction consists in linking every corner $c$ with its successor $s(c)$ by an arc, in a non-crossing fashion. The embedded graph with vertex-set $V(\mathfrak{t}) \cup\left\{v^{\bullet}\right\}$ and edge-set the set of added arcs is then a quadrangulation, rooted according to some convention. See Figure 4. To see that $\mathfrak{q}$ is a quadrangulation, consider an edge of $\mathfrak{t}$ and observe that the face of $\mathfrak{q}$ containing it is indeed of degree 4 by construction.

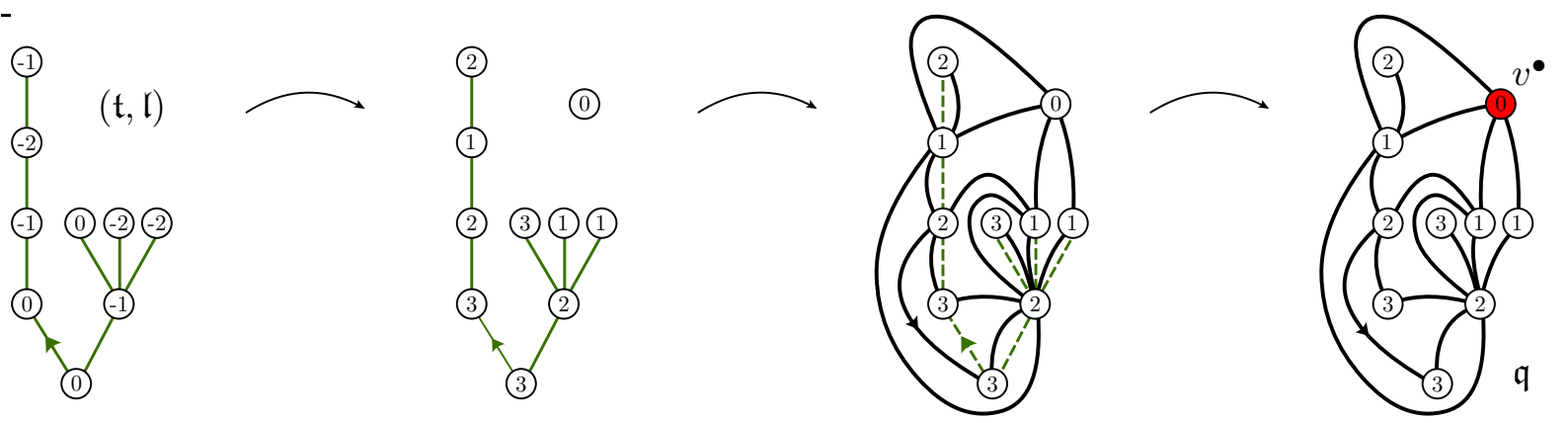

FiguRE 4. Reverse construction. We first translated the labels in such a way that the minimum label equals 0 in order to recover the labels from the previous section. This step does not influence the construction.

The previous constructions are respectively 2-to- 1 and 1-to-2, and are reverse one from another. The factor 2 comes from the rooting convention: the two quadrangulations obtained by reversing the root correspond to the same well-labeled tree.

\subsection{The Brownian snake}

The first step in the comprehension of scaling limits of random maps is often the comprehension of the scaling limits of the encoding objects. In the case of a uniform plane quadrangulation with $n$ faces, the encoding object is a uniform well-labeled tree with $n$ edges and the scaling limit of such an object is a function of the Brownian snake, introduced by Le Gall [LG99]. 
First, let $\mathbf{e}=\left(\mathbf{e}_{t}\right)_{0 \leq t \leq 1}$ be a normalized Brownian excursion (it can for instance be obtained by normalizing the excursion including time 1 of a reflected standard Brownian motion). Then, the so-called Brownian snake's head driven by e may be defined as the process $\left(\mathbf{e}_{t}, Z_{t}\right)_{0 \leq t \leq 1}$, where, conditionally given $\mathbf{e}$, the process $Z$ is a centered Gaussian process with covariance function

$$
\operatorname{Cov}\left(Z_{s}, Z_{t}\right)=\inf _{s \wedge t \leq u \leq s \vee t} \mathbf{e}_{u}
$$

Let us encode a well-labeled tree $(\mathfrak{t}, \mathfrak{l})$ with $n$ edges by its so-called contour and label functions $C$ and $L$ defined as follows. First, let $\mathfrak{t}(0), \mathfrak{t}(1), \ldots, \mathfrak{t}(2 n)$ be the vertices of $\mathfrak{t}$ read in clockwise order around the tree, starting at the root corner. The contour function $C:[0,2 n] \rightarrow \mathbb{R}_{+}$and the label function $L:[0,2 n] \rightarrow \mathbb{R}$ are defined by

$$
C(i):=d_{\mathfrak{t}}(\mathfrak{t}(0), \mathfrak{t}(i)) \quad \text { and } \quad L(i):=\mathfrak{l}(\mathfrak{t}(i)), \quad 0 \leq i \leq 2 n,
$$

and linearly interpolated between integer values (see Figure 5).
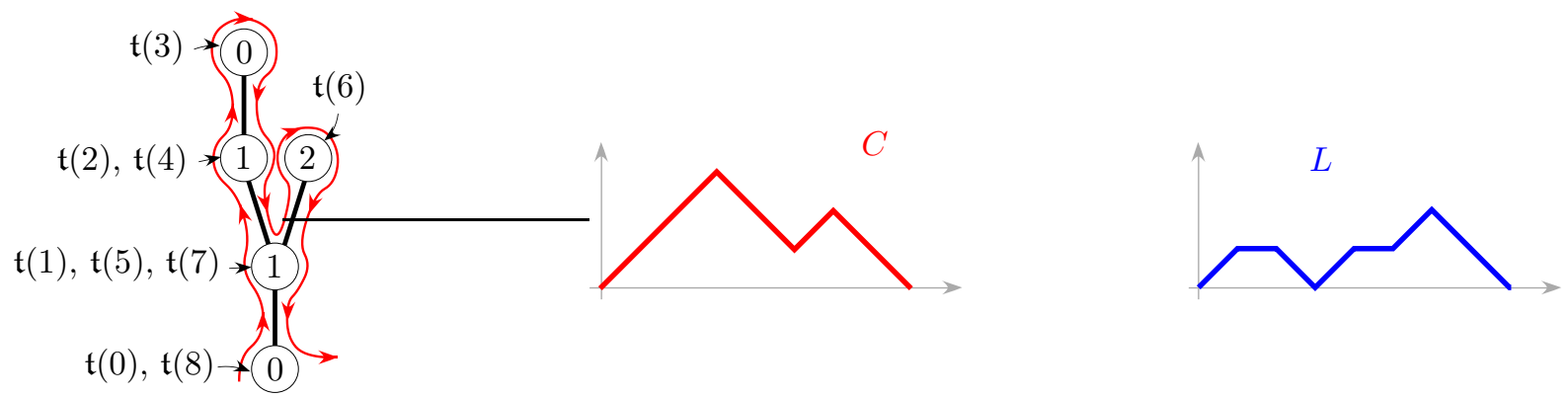

FIgURE 5. The contour and label function associated with a well-labeled tree having 4 edges.

Theorem 6 (Chassaing-Schaeffer [CS04]). Let $C_{n}$ and $L_{n}$ be the contour and label functions associated with a uniform well-labeled tree having $n$ edges. Then

$$
\left(\left(\frac{1}{2 n}\right)^{1 / 2} C_{n}(2 n t),\left(\frac{9}{8 n}\right)^{1 / 4} L_{n}(2 n t)\right)_{0 \leq t \leq 1} \underset{n \rightarrow \infty}{\stackrel{(d)}{\longrightarrow}}\left(\mathbf{e}_{t}, Z_{t}\right)_{0 \leq t \leq 1}
$$

for the uniform topology on $\mathcal{C}\left([0,1], \mathbb{R}^{2}\right)$.

The idea is roughly the following. The convergence of the first coordinate is a conditioned version of Donsker's theorem, which is due to Kaigh [Kai76]. Then, conditionally given the tree $\mathfrak{t}_{n}$, the labels are given by i.i.d. uniform random variables on $\{-1,0,1\}$, which have variance equal to $2 / 3$. As a result, the label variation between times $i$ and $j>i$ is given by a random walk of length $d_{\mathfrak{t}_{n}}(\mathfrak{t}(i), \mathfrak{t}(j))$, which is of order $\sqrt{2 n}\left(\mathbf{e}_{i / 2 n}+\mathbf{e}_{j / 2 n}-\right.$ $\left.2 \min _{i \leq k \leq j} \mathbf{e}_{k / 2 n}\right)$ as a consequence of the convergence of the first coordinate. Donsker's theorem yields that this random walk approximates in the scale $(9 / 8 n)^{1 / 4}$ a Brownian motion of length $\mathbf{e}_{i / 2 n}+\mathbf{e}_{j / 2 n}-2 \min _{i \leq k \leq j} \mathbf{e}_{k / 2 n}$.

We end this section by defining the one-dimensional ISE (random) probability measure $\mu_{\text {ISE }}$ by

$$
\mu_{\mathrm{ISE}}(h):=\int_{0}^{1} d t h\left(Z_{t}\right)
$$

for every nonnegative measurable function $h$. ISE stands for Integrated Super-Brownian Excursion (see [LG99] for more information). This measure is a fundamental object in the study of the Brownian snake and will be used during Section 4. 


\subsection{Construction of the Brownian map}

We may now give an alternative description of the Brownian map, which can be thought of as a continuous version of the Cori-Vauquelin-Schaeffer bijection. Let $\left(\mathbf{e}_{t}, Z_{t}\right)_{0 \leq t \leq 1}$ be the process introduced in the previous section. We first define two pseudo-metrics on $[0,1]$ by

$$
d_{\mathbf{e}}(s, t):=\mathbf{e}_{s}+\mathbf{e}_{t}-2 \min _{s \wedge t \leq r \leq s \vee t} \mathbf{e}_{r} \quad \text { and } \quad d_{Z}(s, t)=Z_{s}+Z_{t}-2 \max \left(\check{Z}_{s, t}, \check{Z}_{t, s}\right),
$$

where $\check{Z}_{s, t}:=\min _{s \leq r \leq t} Z_{r}$ if $s \leq t$ and $\check{Z}_{s, t}:=\min _{r \in[s, 1] \cup[0, t]} Z_{r}$ otherwise. We let $D$ be the largest pseudometric $d$ on $[0,1]$ such that

$$
\left\{d_{\mathbf{e}}=0\right\} \subseteq\{d=0\} \quad \text { and } \quad d \leq d_{Z} .
$$

The Brownian map $(M, D)$ is the quotient space $M:=[0,1] /\{D=0\}$, endowed with the metric induced by $D$ on this set, which we still denote by $D$. Although not obvious at all, it turns out that $D(s, t)=0$ if and only if $d_{\mathbf{e}}(s, t)=0$ or $d_{Z}(s, t)=0$.

If we proceed in two steps and first quotient by $\left\{d_{\mathbf{e}}=0\right\}$ we obtain Aldous's famous Brownian CRT $\mathcal{T}_{\mathbf{e}}:=$ $[0,1] /\left\{d_{\mathbf{e}}=0\right\}$, which arises as the scaling limit of many models of random trees [Ald91]. It is then not very hard to see from Theorem 6 that $d_{\mathbf{e}}(s, t)=0 \Longrightarrow Z_{s}=Z_{t}$ so that we may define a labeling function directly on $\mathcal{T}_{\mathbf{e}}$ by setting, for $a \in \mathcal{T}_{\mathbf{e}}, Z_{a}:=Z_{s}$ where $s \in[0,1]$ is a representative of $a$. Then $M$ is obtained by identifying the points of $\mathcal{T}_{\mathbf{e}}$ having the same label and such that the labels visited inbetween when traveling around the tree are larger than the common label.

\subsection{Local limits}

In the previous sections, we rescaled the metric in order to obtain a compact metric space at the limit. We now completely change our point of view and consider local limits, that is, limits without rescaling, in a topology focusing on the vicinity of the root. This point of view has been widely studied and gives related but quite different objects. In particular, the objects we obtain are noncompact and discrete.

In this setting, both triangulations and quadrangulations are often studied. Let $\mathfrak{t}_{n}$ be a triangulation chosen uniformly at random among all triangulations with $n$ vertices. Angel and Schramm [AS03] have introduced an infinite random plane triangulation $T_{\infty}$, called the Uniform Infinite plane Triangulation (UIPT), which is obtained as the local limit of $t_{n}$ as $n \rightarrow \infty$. More precisely, $T_{\infty}$ is characterized by the fact that, for every $r \geq 0$, we have the following convergence in distribution

$$
B_{r}\left(\mathfrak{t}_{n}\right) \underset{n \rightarrow \infty}{\stackrel{(d)}{\longrightarrow}} B_{r}\left(T_{\infty}\right),
$$

where $B_{r}(\mathfrak{m})$ denotes the map formed by the edges and vertices of the map $\mathfrak{m}$ lying at graph distance smaller than or equal to $r$ from the origin of the root. This infinite random triangulation and its quadrangulation analog (the UIPQ, see [CD06, Kri05]) have attracted a lot of attention, see [BC13, Cur13, GGN13] and the references therein. We will also come back to this object in Section 3, where percolation on it is studied.

Note that the scaling factor appearing in the results of Section 1.1 is always $n^{-1 / 4}$ and, in this section, we did not rescale the metric. One might wonder what happens if one rescales by a different factor, say $n^{-\alpha}$ for some $\alpha \in(0,1 / 4)$. The resulting object is called the Brownian plane and was introduced by Curien and Le Gall [CLG12]. This object may also be obtained by scaling the metric of the UIPQ by a factor $\lambda$ and by letting $\lambda \rightarrow 0$.

The subsequent sections summarize the original works presented during the random maps session of the Journées MAS 2014. 


\section{Rescaled bipartite plane maps converge to the Brownian map [By C. ABRAHAM]}

The work [Abr13] is an addition to a series of papers that focus on the convergence of large random plane maps viewed as metric spaces to the Brownian map. The main goal is to provide another example of such limit theorems, in the case of bipartite plane maps with a fixed number of edges.

Recall that a map is bipartite if its vertices can be colored with two colors in such a way that any two vertices that have the same color are not connected by any edge. Equivalently, a map is bipartite if and only if all the cycles it contains have an even length. In the case of plane maps, this boils down to the condition that all the faces are of even degree. Indeed, the Jordan curve theorem implies that a cycle always separates a plane map into two plane submaps (corresponding to the two connected components). Observing that every edge is incident to exactly two faces, we see that the sum of the degrees of the faces is even, so that the number of odd degree-faces is even. As a result, if a plane map contains a cycle of odd length, both submaps it separates contain an odd number of odd degree-faces, hence at least one.

Theorem 7 (Abraham [Abr13]). For every $n \geq 1$, let $\mathfrak{m}_{n}$ be uniformly distributed over the set of bipartite plane maps with $n$ edges. Then, the following convergence holds in distribution for the Gromov-Hausdorff topology,

$$
\left(V\left(\mathfrak{m}_{n}\right),(2 n)^{-1 / 4} d_{\mathfrak{m}_{n}}\right) \underset{n \rightarrow \infty}{\stackrel{(d)}{\longrightarrow}}(M, D)
$$

where $(M, D)$ is the Brownian map introduced during Section 1.1.

Let us compare this theorem with a similar result obtained by Bettinelli, Jacob and Miermont around the same time, using a different method.

Theorem 8 (Bettinelli-Jacob-Miermont [BJM14]). For every $n \geq 1$, let $\mathfrak{m}_{n}^{\prime}$ be uniformly distributed over the set of plane maps with $n$ edges. Then, the following convergence holds in distribution for the Gromov-Hausdorff topology,

$$
\left(V\left(\mathfrak{m}_{n}^{\prime}\right),\left(\frac{9}{8 n}\right)^{1 / 4} d_{\mathfrak{m}_{n}^{\prime}}\right) \underset{n \rightarrow \infty}{\stackrel{(d)}{\longrightarrow}}(M, D) .
$$

These two theorems are almost identical, the difference being the word "bipartite" and the value of the normalizing constant: $2^{-1 / 4}$ in the bipartite case and $(9 / 8)^{1 / 4}$ in the general case. This means that, from a metric point of view, a large uniform bipartite plane map looks very much like a uniform plane map rescaled by the factor $\sqrt{3 / 2} \approx 1.22$. Heuristically, imposing that the map be bipartite forces its faces to be of larger degree and thus produces larger distances. A similar phenomenon appears if we compare uniform simple triangulations/quadrangulations with uniform triangulations/quadrangulations without restrictions. In these cases, the scaling constant is $\sqrt{2} \approx 1.41$ for triangulations and $3^{1 / 4} \approx 1.32$ for quadrangulations.

Note that, in contrast with Theorem 2, we considered in both theorems maps with a given number of edges instead of a given number of faces. This is because there exist infinitely many bipartite plane maps with any fixed number of faces (one can for instance add two vertices on an edge of a bipartite plane and obtain another bipartite plane map with the same number of faces). Similarly, there exist infinitely many bipartite plane maps with any given number of vertices (one might for instance double an edge). As a result, the only natural choice is to condition on the number of edges. Unfortunately, such a conditioning is not as nicely behaved on the encoding objects and results in certain additional technical difficulties.

The proof of Theorem 7 relies on a generalization of the combinatorial bijection presented in Section 1.3, which is due to Bouttier, Di Francesco and Guitter [BD G04]. It specializes into a bijection between rooted and pointed bipartite maps and certain two-type labeled trees. The random labeled tree associated with a uniform rooted and pointed bipartite map with $n$ edges via this bijection is a labeled two-type Galton-Watson tree with explicit offspring distributions, conditioned to have a fixed progeny $n$. More precisely, vertices of the first type are labeled and correspond to the vertices of the map, with the exception of the distinguished one (as in 
the Cori-Vauquelin-Schaeffer bijection) and the vertices of the other type encode the faces of the map. For instance, the degree of a vertex of the second type is half the degree of the corresponding face.

Let us denote by $\left(T_{n}, \ell_{n}\right)$ this labeled two-type tree, where $\ell_{n}$ is an integer-valued function defined on a subset $T_{n}^{\circ} \subseteq V\left(T_{n}\right)$ containing the root vertex. In order to prove the convergence to the Brownian map, an important technical step is to obtain an analog of Theorem 6 , that is, the limit of the contour and label functions associated with $\left(T_{n}, \ell_{n}\right)$. As the labels are only set on the vertex-subset $T_{n}^{\circ}$, we slightly modify the definition of the contour and label functions. We let $T_{n}^{\circ}(0), T_{n}^{\circ}(1), \ldots, T_{n}^{\circ}(n)$ denote the vertices of $T_{n}^{\circ}$ read in clockwise order around the tree, starting at the (labeled) root corner. We then set

$$
C_{n}^{\circ}(i):=d_{T_{n}}\left(T_{n}^{\circ}(0), T_{n}^{\circ}(i)\right) / 2 \quad \text { and } \quad L_{n}^{\circ}(i):=\mathfrak{l}\left(T_{n}^{\circ}(i)\right), \quad 0 \leq i \leq n
$$

and extend the definition to $[0, n]$ by linear interpolation. Recall that $(\mathbf{e}, Z)$ was introduced during Section 1.4.

Theorem 9 (Abraham [Abr13]). The following convergence

$$
\left(\left(\frac{81}{32 n}\right)^{1 / 2} C_{n}^{\circ}(n t),(2 n)^{-1 / 4} L_{n}^{\circ}(n t)\right)_{0 \leq t \leq 1} \underset{n \rightarrow \infty}{\stackrel{(d)}{\longrightarrow}}\left(\mathbf{e}_{t}, Z_{t}\right)_{0 \leq t \leq 1}
$$

holds for the uniform topology on $\mathcal{C}\left([0,1], \mathbb{R}^{2}\right)$.

The fact that the tree is conditioned here on having a fixed number of vertices rather than a fixed number of vertices of a given type is non standard, This creates an additional difficulty, which we handle through an absolute continuity argument. A useful technical ingredient is a seemingly new definition of a "modified" Lukasiewicz path associated with a two-type tree.

Another difficulty arises from the fact that the encoding bijection uses pointed maps. Indeed, recall that $\left(T_{n}, \ell_{n}\right)$ was the labeled two-type tree associated with a uniform pointed bipartite map with $n$ edges. As the number of vertices in a uniform bipartite map with $n$ edges is random, uniformly pointing a uniform bipartite map with $n$ edges does not yield a uniform pointed bipartite map with $n$ edges. We then see a bias to the distribution of $\left(T_{n}, \ell_{n}\right)$. We manage to overcome this difficulty at the cost of a technical "depointing" lemma, relying on the fact that the number of vertices, although random, is asymptotically concentrated around a deterministic number.

Note that the previous difficulty did not occur in the case of quadrangulations as the number of vertices of a quadrangulation with $n$ faces only depends on $n$ (it is equal to $n+2$ by the Euler characteristic formula).

\section{Percolation on random triangulations \& looptrees [By I. Kortchemski]}

The work [CK14] studies site percolation on Angel \& Schramm's UIPT (see Section 1.6). Its goal is to compute several critical and near-critical exponents, and describe the scaling limit of the boundary of large percolation clusters in all regimes (subcritical, critical and supercritical). One of the contributions of [CK14] is to prove in particular that the scaling limit of the boundary of large critical percolation clusters is the random stable looptree of index $3 / 2$, which was introduced in [CK13].

The goal of the probabilistic theory of random plane maps is to understand universal large-scale properties of random plane graphs or maps. It is a very active field of research, along with its physics counterpart, the Liouville 2D quantum gravity, whose goal is, very roughly speaking, to study a surface chosen "uniformly at random" (whose discretization is modeled by a random map), possibly coupled with matter (meaning that the random map is decorated with a statistical physics matter), and to study the influence of this coupling through the celebrated KPZ formula (see [DKRV14,DS11]).

In [CK14], Curien and Kortchemski focus on one of the simplest models of (critical) statistical mechanics models on random lattices: site percolation on the UIPT, which we now introduce.

Percolation on the UIPT. Given the UIPT, we consider a site percolation by coloring its vertices independently white with probability $a \in(0,1)$ and black with probability $1-a$. This model has already been studied 
by Angel [Ang03], who proved that the critical threshold is almost surely equal to

$$
a_{c}=1 / 2
$$

His approach was based on a clever Markovian exploration of the UIPT called the peeling process. See also [AC13, Cur13, MN14] for further studies of percolation on random maps using the peeling process.

In [CK14], the authors are interested in the geometry of the boundary of percolation clusters and use a different approach. We condition the root edge of the UIPT on being of the form $\circ \rightarrow \bullet$, which will allow us to define the percolation interface going through the root edge. The white cluster of the origin is by definition the set of all the white vertices and edges between them that can be reached from the origin of the root edge using white vertices only. We denote by $\mathcal{H}_{a}^{\circ}$ its hull, which is obtained by filling-in the holes of the white cluster except the one containing the target of the root edge called the exterior component (see Figure 6). Finally, we denote by $\partial \mathcal{H}_{a}^{\circ}$ the boundary of the hull, which is the graph formed by the edges and vertices of $\mathcal{H}_{a}^{\circ}$ adjacent to the exterior (see Figure 6), and let $\# \partial \mathcal{H}_{a}^{\circ}$ be its perimeter, or length, that is the number of oriented edges of $\partial \mathcal{H}_{a}^{\circ}$ belonging to the exterior. Note that $\partial \mathcal{H}_{a}^{\circ}$ is formed of discrete cycles attached by some pinch-points. It follows from the work of Angel [Ang03] that, for every value of $a \in(0,1)$, the boundary $\partial \mathcal{H}_{a}^{\circ}$ is always finite (if an infinite interface separating a black cluster from a white cluster existed, this would imply the existence of both infinite black and white clusters, which is intuitively not posible).

One of the contributions of [CK14] is to find the precise asymptotic behavior for the probability of having a large perimeter in the critical case.
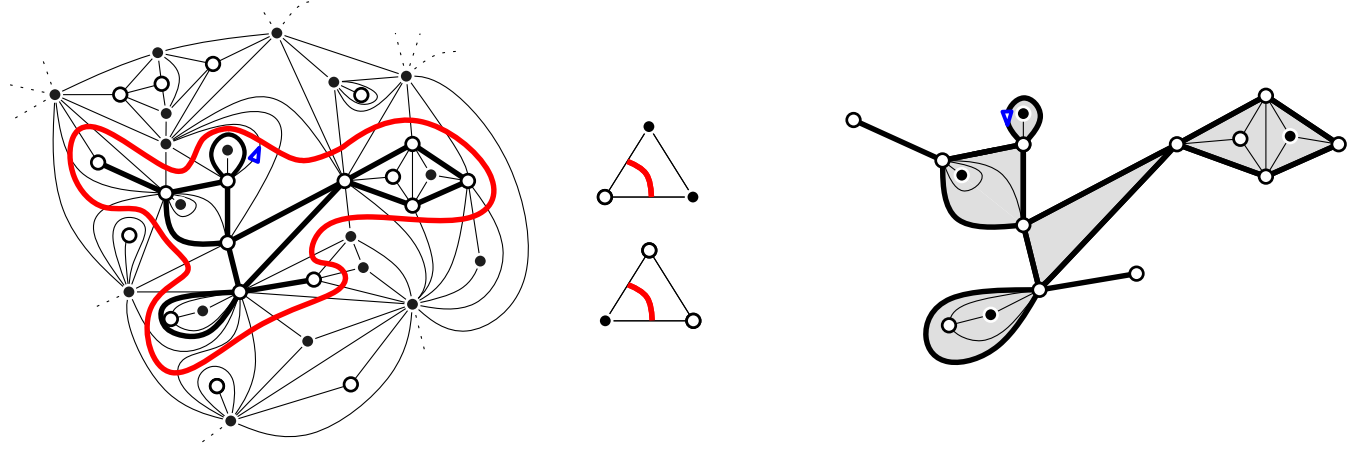

FIGURE 6. On the left, a part of a site-percolated triangulation with the interface going through the root edge and the hull of the cluster of the origin. The interface is drawn using the rules displayed in the middle triangles. On the right, the boundary of the hull is in bold black line segments and has perimeter 16.

Theorem 10 (Curien-Kortchemski [CK14]). Critical exponent for the perimeter.

For $a=a_{c}=1 / 2$ we have

$$
\mathbb{P}\left(\# \partial \mathcal{H}_{\frac{1}{2}}^{\circ}=n\right) \underset{n \rightarrow \infty}{\sim} \frac{3}{2 \cdot|\Gamma(-2 / 3)|^{3}} \cdot n^{-4 / 3},
$$

where $\Gamma$ is Euler's Gamma function.

It is interesting to mention that the exponent $4 / 3$ for the perimeter of the boundary of critical clusters also appears in [AC13], where it is shown that $\mathbb{P}\left(\# \partial \mathcal{H}_{1 / 2}^{\circ}>n\right) \asymp n^{-1 / 3}$, where $a_{n} \asymp b_{n}$ means that the sequence $a_{n} / b_{n}$ is bounded from below and above by certain constants. Actually, this reference focuses on the UIPT of 
the upper half plane, which is a different, but similar model, and uses a different approach approach based on the so-called peeling process, which is a mean to iteratively explore the map in a Markovian way.

One of the techniques used to establish Theorem 10 is a surgery operation inspired from Borot, Bouttier \& Guitter [BBG12b,BBG12a]. This allows the authors to derive a tree representation of the 2-connected components of $\partial \mathcal{H}_{a}^{\circ}$, which is proved to be closely related to the law of a certain two-type Galton-Watson tree. The study of this two-type random tree is reduced to the study of a standard one-type Galton-Watson tree by using a recent bijection due to Janson \& Stefánsson [JS12], which enables the use of the vast literature on random trees and branching processes to make exact computations.

This method also allows to fully understand the probabilistic structure of the hull of the white cluster and to identify the scaling limits (for the Gromov-Hausdorff topology) in any regime (subcritical, critical and supercritical) of $\partial \mathcal{H}_{a}^{\circ}$, seen as a compact metric space, when its perimeter tends to infinity. In particular, the authors establish that the scaling limit of $\partial \mathcal{H}_{a_{c}}^{\circ}$ conditioned to be large, appropriately rescaled, is the stable looptree of parameter $3 / 2$ introduced in [CK13], whose definition we now recall.

Stable looptrees. Random stable looptrees are random compact metric spaces and can, in a certain sense, be seen as the dual of the stable trees introduced and studied in [DLG02,LGLJ98]. They are constructed in [CK13] using stable processes with no negative jumps, but can also be defined as scaling limits of discrete objects: with every rooted oriented tree (or plane tree) $\tau$, we associate a graph, called the discrete looptree of $\tau$ and denoted by $\operatorname{Loop}(\tau)$, which is the graph on the set of vertices of $\tau$ such that two vertices $u$ and $v$ are joined by an edge if and only if one of the following three conditions are satisfied in $\tau: u$ and $v$ are consecutive children of a same parent, or $u$ is the first child (in the lexicographical order) of $v$, or $u$ is the last child of $v$, see Figure 7 . Note that in [CK13], $\operatorname{Loop}(\tau)$ is defined as a different graph, and that here $\operatorname{Loop}(\tau)$ is the graph which is denoted by $\operatorname{Loop}^{\prime}(\tau)$ in [CK13]. We view $\operatorname{Loop}(\tau)$ as a compact metric space by endowing its vertex-set with the graph metric (every edge has unit length).
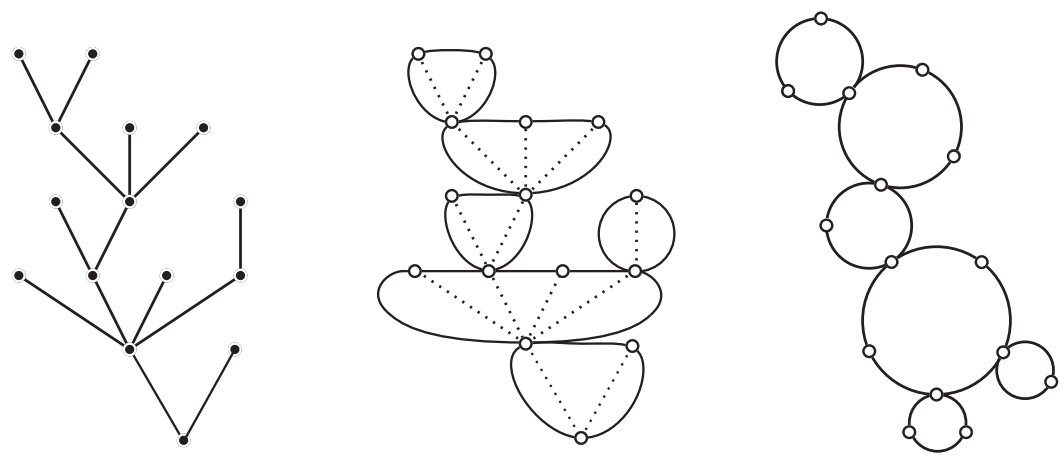

Figure 7. A plane tree $\tau$ (left) and its looptree Loop $(\tau)$ (middle and right).

Fix $\alpha \in(1,2)$. Now let $\tau_{n}$ be a Galton-Watson tree conditioned on having $n$ vertices, whose offspring distribution $\mu$ is critical and satisfies $\mu_{k} \sim c \cdot k^{-1-\alpha}$ as $k \rightarrow \infty$ for a certain $c>0$. In [CK13, Section 4.2], it is shown that there exists a random compact metric space $\mathscr{L}_{\alpha}$, called the stable looptree of index $\alpha$, such that

$$
n^{-1 / \alpha} \cdot \operatorname{Loop}\left(\tau_{n}\right) \underset{n \rightarrow \infty}{\stackrel{(d)}{\longrightarrow}}(c|\Gamma(-\alpha)|)^{-1 / \alpha} \cdot \mathscr{L}_{\alpha}
$$

where the convergence holds in distribution for the Gromov-Hausdorff topology and where $c \cdot M$ stands for the metric space obtained from $M$ by multiplying all distances by $c>0$.

It has been proved in [CK13] that the Hausdorff dimension of $\mathscr{L}_{\alpha}$ is almost surely equal to $\alpha$. Furthermore, the stable looptrees can be seen as random metric spaces interpolating between the unit length circle $\mathcal{C}_{1}:=$ 


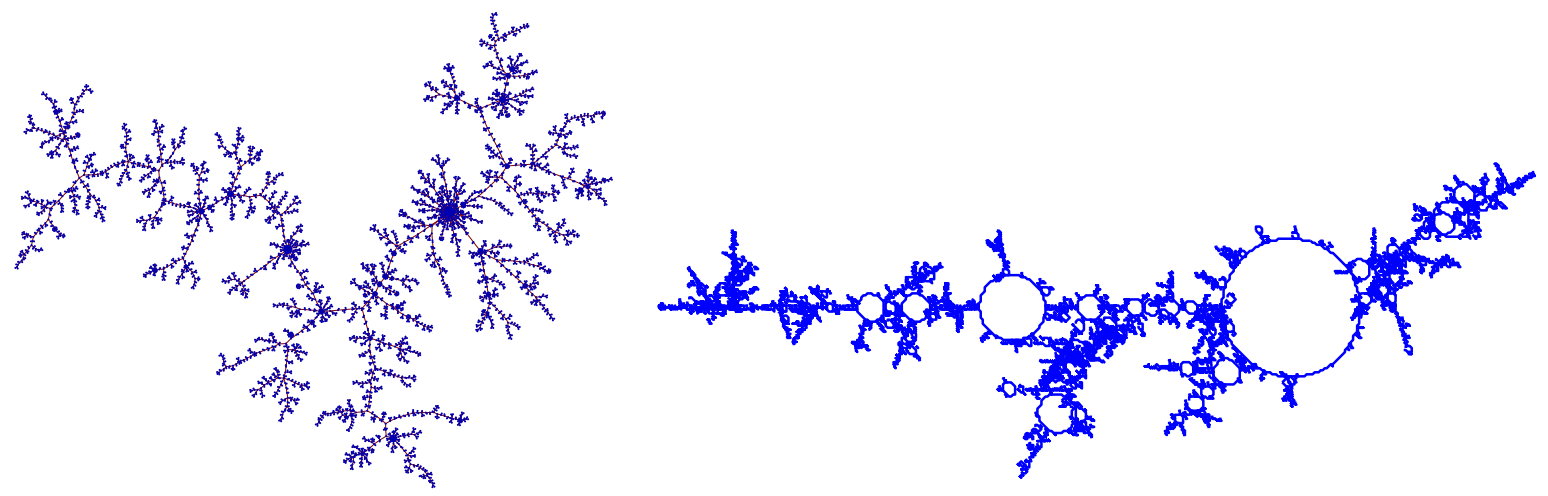

Figure 8. An $\alpha=3 / 2$ stable tree, and its associated looptree $\mathscr{L}_{3 / 2}$, embedded in a non isometric and non proper fashion in the plane.

$\frac{1}{2 \pi} \cdot \mathbb{S}_{1}$ (when $\alpha \downarrow 1$ ) and Aldous's Brownian CRT [Ald91] (when $\alpha \uparrow 2$ ), which we view here as the real tree $\mathcal{T}_{\mathbf{e}}:=\left([0,1] /\left\{d_{\mathbf{e}}=0\right\}, d_{\mathbf{e}}\right.$ ) with the notation of Section 1.5 (see [LG05]). We are now in position to describe the possible scaling limits of the boundary of percolation clusters in the UIPT. For fixed $a \in(0,1)$, let $\partial \mathcal{H}_{a}^{\circ}(n)$ be the boundary of the white hull of the origin conditioned on the event that $\mathcal{H}_{a}^{\circ}$ is finite and that the perimeter of $\partial \mathcal{H}_{a}^{\circ}$ is $n$. We view $\partial \mathcal{H}_{a}^{\circ}(n)$ as a compact metric space by endowing its vertex-set with the graph metric.

Theorem 11 (Curien-Kortchemski [CK14]). Scaling limits for $\partial \mathcal{H}_{a}^{\circ}$ when $\# \mathcal{H}_{a}^{\circ}<\infty$.

For every $a \in(0,1)$, there exists a positive constant $C_{a}$ such that the following convergences hold in distribution for the Gromov-Hausdorff topology:

$$
\begin{array}{llll}
\text { (i) } \quad \text { when } 1 / 2<a<1, & n^{-1} \cdot \partial \mathcal{H}_{a}^{\circ}(n) & \underset{n \rightarrow \infty}{\stackrel{(d)}{\longrightarrow}} & C_{a} \cdot \mathcal{C}_{1}, \\
\text { (ii) } \quad \text { when } a=a_{c}=1 / 2, & n^{-2 / 3} \cdot \partial \mathcal{H}_{a}^{\circ}(n) & \underset{n \rightarrow \infty}{\stackrel{(d)}{\longrightarrow}} & 3^{1 / 3} \cdot \mathscr{L}_{3 / 2}, \\
\text { (iii) } \quad \text { when } 0<a<1 / 2, & n^{-1 / 2} \cdot \partial \mathcal{H}_{a}^{\circ}(n) & \underset{n \rightarrow \infty}{\stackrel{(d)}{\longrightarrow}} & C_{a} \cdot \mathcal{T}_{\mathbf{e}} .
\end{array}
$$

Although Theorem 11 does not imply that $1 / 2$ is the critical threshold for percolation on the UIPT (as shown in [Ang03]), it is a compelling evidence for it. Let us give a heuristic justification for the three limiting compact metric spaces appearing in the statement of this theorem. Imagine that we condition the cluster of the origin to be finite and have a very large, but finite, boundary. In the supercritical regime $(i)$, as soon as the cluster grows arms it is likely to become infinite, hence the easiest way to stay finite is to look like a loop. On the contrary, in the subcritical regime (iii), having a large boundary costs a lot, so the cluster adopts the shape which maximizes its boundary length for fixed size: the tree. In the critical case $(i i)$, these effects are balanced and a fractal object emerges: not quite a loop, nor a tree, but a looptree!

Also, the proof of Theorem 11 gives the expression of $C_{a}$ in terms of certain quantities involving GaltonWatson trees, which allows to obtain the near-critical scaling behaviors of $C_{a}$ as $a \uparrow 1 / 2$ and $a \downarrow 1 / 2$.

Let us mention that the exponents appearing in the previous theorems are expected to be universal, and it is believed that the techniques of [CK14] may be extended to prove that the stable looptrees $\left(\mathscr{L}_{\alpha}: \alpha \in(1,2)\right)$ give the scaling limits of the outer boundary of clusters of suitable statistical mechanics models on random plane triangulations. 


\section{On the distance-Profile of rooted simple maps [By G. Collet]}

In [BCF14], we prove the convergence of the distance-profile for rooted simple maps, that is, plane maps with neither loops nor multiple edges. We also show that it implies the same type of result for the class of loopless maps, and for the class of all plane maps. We now give a few definitions in view of stating our main result. For a rooted plane map $\mathfrak{m}$ with $n$ edges, the distance $d(e)$ of an edge $e$ (with respect to the root) is the length of a shortest path starting at (an extremity of) $e$ and ending at the root-vertex; the distance-profile of $\mathfrak{m}$ is the $n$-set $\{d(e)\}_{e \in E}$, where $E$ denotes the edge-set of $\mathfrak{m}$. (Notice that we consider a distance-profile at edges, not at vertices.) Let us now give some terminology for the type of convergence results to be obtained. We denote by $\mathcal{M}_{1}$ the set of probability measures on $\mathbb{R}$, endowed with the weak topology (that is, the topology given by the convergence in law). For $\mu \in \mathcal{M}_{1}$, we denote by $F_{\mu}(x)$ the cumulative function of $\mu$,

$$
\inf (\mu):=\inf \left\{x: F_{\mu}(x)>0\right\} \quad \text { and } \quad \sup (\mu):=\sup \left\{x: F_{\mu}(x)<1\right\},
$$

and we define the width of $\mu$ as $\sup (\mu)-\inf (\mu)$. We also define the nonnegative shift of $\mu$ as the probability measure (with support in $\mathbb{R}_{+}$) whose cumulative function is $x \mapsto F_{\mu}(x+\inf (\mu))$.

Definition 1. We let $\mu_{\mathrm{ISE}}$ be a random variable with ISE law (as defined at the end of Section 1.4), and $\mu_{\mathrm{ISE}}^{\mathrm{shift}}$ be its non-negative shift; these are random variables taking values in $\mathcal{M}_{1}$. A sequence $\mu^{(n)}$ of random variables taking values in $\mathcal{M}_{1}$ is said to satisfy the ISE limit property if the following properties hold:

- $\mu^{(n)}$ converges in law to $\mu_{\mathrm{ISE}}^{\mathrm{shift}}$ (for the weak topology on $\mathcal{M}_{1}$ ).

- $\sup \left(\mu^{(n)}\right)$ converges in law to $\sup \left(\mu_{\mathrm{ISE}}^{\mathrm{shift}}\right)$ (i.e., the width of $\left.\mu_{\mathrm{ISE}}\right)$.

It is easy to see that if a sequence $\mu^{(n)}$ of random variables taking values in $\mathcal{M}_{1}$ converges in law to $\mu \in$ $\mathcal{M}_{1}$, then a real random variable with distribution $\mu^{(n)}$ converges in law toward a real random variable with distribution $\mu$. It is known that a real random variable with distribution $\mu_{\mathrm{ISE}}^{\text {shift }}$ is distributed as $\sup \left(\mu_{\mathrm{ISE}}\right)($ whose cumulative function has an explicit expression, see [BDFG03]). Hence, if $\mu^{(n)}$ has the ISE limit property, then a real random variable with distribution $\mu^{(n)}$ converges in law toward $\sup \left(\mu_{\mathrm{ISE}}\right)$.

For an $n$-set $\mathbf{x}=\left\{x_{1}, \ldots, x_{n}\right\}$ of nonnegative values, and for $a>0$, define $\mu_{a}(\mathbf{x})$ as the probability measure

$$
\mu_{a}(\mathbf{x})=\frac{1}{n} \sum_{i=1}^{n} \delta_{x_{i} /(a n)^{1 / 4}},
$$

where $\delta_{x}$ denotes the Dirac measure at $x$. Our main result is the following:

Theorem 12 (Bernardi-Collet-Fusy [BCF14]). For $n \geq 1$, let $\pi_{n}^{(\mathrm{s})}$ be the distance-profile of a uniformly random rooted simple map with $n$ edges. Then $\mu_{2}\left(\pi_{n}^{(\mathrm{s})}\right)$ satisfies the ISE limit property.

The proof of Theorem 12 relies on a bijection between a subfamily of simple maps with a root face of degree 3 (named outertriangular simple maps) and well-known Eulerian triangulations (triangulations whose vertices have even degree). For any outertriangular simple map, there exists a canonical way (using local rules) to orient its inner edges and add some additionnal outgoing half-edges at its vertices: this orientation is called the canonical 3-orientation with buds. Such orientations play a crucial part in combinatorics of maps and in particular in many bijections between maps and trees (which are related to but quite different from the bijection presented in Section 1.3). The terminology comes from the fact that each inner vertex has outdegree 3 , that is, is such that exactly 3 edges or half-edges are oriented away from it. The bijection yields, through "inflation" of the inner edges into triangles, an Eulerian triangulation, which is itself endowed with a given (partial) orientation: its canonical 1-orientation (see Figure 9).

In a rooted map endowed with a "reasonable" orientation such as the two orientations mentioned above, one can define the rightmost path $p(e)$ of an edge $e$ as the oriented path starting at $e$ and following at each step the rightmost outgoing edge, eventually reaching the root. By extending a result of Addario-Berry and Albenque on 


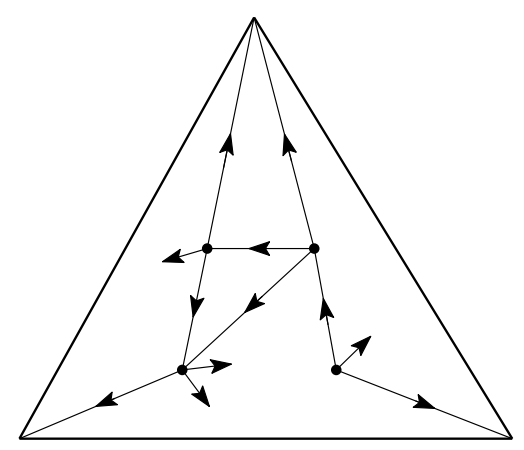

$(a)$

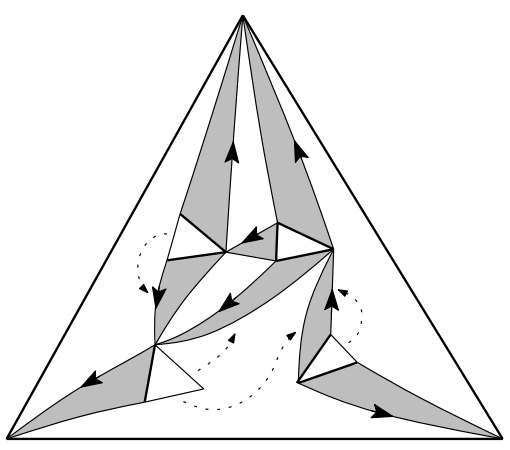

(b)

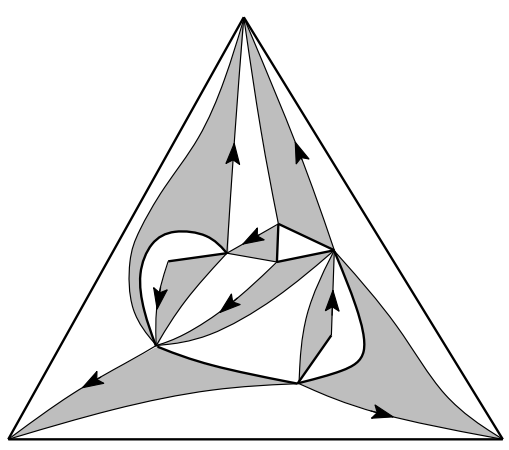

$(c)$

FiguRE 9. The two steps of the bijection between $(a)$ an outertriangular simple map with $n+2$ edges endowed with its canonical 3-orientation, and $(c)$ an Eulerian triangulation with $2 n$ faces endowed with its canonical 1-orientation.

simple triangulations [ABA13], we show that rightmost paths are almost surely "geodesic" - in the sense that, when rescaled by $n^{1 / 4}$, they are close to the shortest path - in random outertriangular simple maps. Rightmost paths are then transported via the bijection on canonical oriented paths in Eulerian triangulations, where the distance-profile (for the oriented metric given by the canonical orientation) has already been proved to satisfy the ISE limit property (see [BDG04] for the encoding of the maps and [LG06] for the convergence). Finally we conclude by exploiting an easy decomposition of simple maps into a sequence of outertriangular simple maps: almost surely there exists a "giant outertriangular component" where the diameter is concentrated. We can then transfer the previous result to simple maps.

It has been shown in [GW99, BFSS01] that when $n$ gets large, a uniformly random rooted loopless map of size $n$ has almost surely a unique "giant component" $B$, which is a uniformly random simple map whose size is concentrated around $2 n / 3$, and the second largest component has size $O\left(n^{2 / 3+\delta}\right)$ for any $\delta>0$. It follows that a loopless map can be described as its largest simple component $B$ (almost surely of diameter $O\left((2 \cdot 2 n / 3)^{1 / 4}\right)=O\left((4 n / 3)^{1 / 4}\right)$ by Theorem 12), whose edges are substituted by "small" loopless components, each almost surely of diameter at most $O\left(n^{7 / 32}\right)$ (thanks to an exponential decay property satisfied by the diameter). Hence we obtain the following result:

Corollary 13 (Bernardi-Collet-Fusy [BCF14]). For $n \geq 1$, let $\pi_{n}^{(1)}$ be the distance-profile of a uniformly random rooted loopless map with $n$ edges. Then $\mu_{4 / 3}\left(\pi_{n}^{(1)}\right)$ satisfies the ISE limit property.

Similarly, it has been shown in [GW99,BFSS01] that when $n$ gets large, a uniformly random rooted map of size $n$ has almost surely a unique "giant component" $L$, which is a uniformly random loopless map whose size is concentrated around $2 n / 3$, and the second largest component has size $O\left(n^{2 / 3+\delta}\right)$ for any $\delta>0$. One can thus decompose a loopless map as its largest loopless component $L$, where in each corner can be attached "small" general maps, each delimited by a loop. Hence by controlling the diameter of each component, we recover here a known result, which alternatively follows from the study of the profile of random rooted quadrangulations [CS04], combined with the recent profile-preserving bijection in [AB13] between quadrangulations and maps.

Corollary 14 (Bernardi-Collet-Fusy [BCF14]). For $n \geq 1$, let $\pi_{n}^{(\mathrm{g})}$ be the distance-profile of a uniformly random rooted general map with $n$ edges. Then $\mu_{8 / 9}\left(\pi_{n}^{(\mathrm{g})}\right)$ satisfies the ISE limit property.

\section{REFERENCES}

[AB13] Jan Ambjørn and Timothy G. Budd. Trees and spatial topology change in causal dynamical triangulations. J. Phys. A, 46(31):315201, 33, 2013. 
[ABA13] Louigi Addario-Berry and Marie Albenque. The scaling limit of random simple triangulations and random simple quadrangulations. Preprint, arXiv: 1306. 5227, 2013.

[Abr13] Céline Abraham. Rescaled bipartite planar maps converge to the Brownian map. Preprint, arXiv: 1312 . $5959,2013$.

[AC13] Omer Angel and Nicolas Curien. Percolations on infinite random maps i, half-plane models. arXiv: 1301. 5311, to appear in Ann. IHP. Prob. Stat., 2013.

[Ald91] David Aldous. The continuum random tree. I. Ann. Probab., 19(1):1-28, 1991.

[Ang03] Omer Angel. Growth and percolation on the uniform infinite planar triangulation. Geom. Funct. Anal., 13(5):935-974, 2003.

[AS03] Omer Angel and Oded Schramm. Uniform infinite planar triangulations. Comm. Math. Phys., 241(2-3):191-213, 2003.

[BBG12a] Gaetan Borot, Jérémie Bouttier, and Emmanuel Guitter. More on the o $n)$ model on random maps via nested loops: loops with bending energy. J. Phys. A: Math. Theor., 45(27):275206, 2012.

[BBG12b] Gaetan Borot, Jérémie Bouttier, and Emmanuel Guitter. A recursive approach to the $o(n)$ model on random maps via nested loops. J. Phys. A: Math. Theor., 45(4):045002, 2012.

[BBI01] Dmitri Burago, Yuri Burago, and Sergei Ivanov. A course in metric geometry, volume 33 of Graduate Studies in Mathematics. American Mathematical Society, Providence, RI, 2001.

[BC13] Itai Benjamini and Nicolas Curien. Simple random walk on the uniform infinite planar quadrangulation: subdiffusivity via pioneer points. Geom. Funct. Anal., 23(2):501-531, 2013.

[BCF14] Olivier Bernardi, Gwendal Collet, and Éric Fusy. On the distance-profile of random rooted plane graphs. to appear in AofA'14, 2014.

[BDFG03] Jérémie Bouttier, Philippe Di Francesco, and Emmanuel Guitter. Geodesic distance in planar graphs. Nuclear Phys. B, 663(3):535-567, 2003.

[BDG04] Jérémie Bouttier, Philippe Di Francesco, and Emmanuel Guitter. Planar maps as labeled mobiles. Electron. J. Combin., 11(1):Research Paper 69, 27 pp. (electronic), 2004.

[Bet14] Jérémie Bettinelli. Geodesics in Brownian surfaces (Brownian maps). arXiv:1401.3602, 2014.

[BFSS01] Cyril Banderier, Philippe Flajolet, Gilles Schaeffer, and Michèle Soria. Random maps, coalescing saddles, singularity analysis, and Airy phenomena. Random Structures Algorithms, 19(3-4):194-246, 2001. Analysis of algorithms (Krynica Morska, 2000).

[BJM14] Jérémie Bettinelli, Emmanuel Jacob, and Grégory Miermont. The scaling limit of uniform random plane maps, via the Ambjørn-Budd bijection. Electron. J. Probab., 19:no. 74, 1-16, 2014.

[BLG13] Johel Beltran and Jean-François Le Gall. Quadrangulations with no pendant vertices. Bernoulli, 19(4):1150-1175, 2013.

[BM14] Jérémie Bettinelli and Grégory Miermont. Uniqueness of Brownian surfaces by a surgical approach. in preparation, 2014.

[CD06] Philippe Chassaing and Bergfinnur Durhuus. Local limit of labeled trees and expected volume growth in a random quadrangulation. Ann. Probab., 34(3):879-917, 2006.

[CK13] Nicolas Curien and Igor Kortchemski. Random stable looptrees. Preprint, arXiv: 1304. 1044, 2013.

[CK14] Nicolas Curien and Igor Kortchemski. Percolation on random triangulations and stable looptrees. arXiv: 1307. 6818, to appear in Probab. Theor. Rel. Fields., 2014.

[CLG12] Nicolas Curien and Jean-François Le Gall. The brownian plane. Journal of Theoretical Probability, pages 1-43, 2012.

[CMS09] Guillaume Chapuy, Michel Marcus, and Gilles Schaeffer. A bijection for rooted maps on orientable surfaces. SIAM J. Discrete Math., 23(3):1587-1611, 2009.

[CS04] Philippe Chassaing and Gilles Schaeffer. Random planar lattices and integrated superBrownian excursion. Probab. Theory Related Fields, 128(2):161-212, 2004.

[Cur13] Nicolas Curien. A glimpse of the conformal structure of random planar maps. Preprint, arXiv: $1308.1807,2013$.

[CV81] Robert Cori and Bernard Vauquelin. Planar maps are well labeled trees. Canad. J. Math., 33(5):1023-1042, 1981.

[DKRV14] François David, Antti Kupiainen, Rémi Rhodes, and Vincent Vargas. Liouville Quantum Gravity on the Riemann sphere. arXiv: 1410. 7318, 2014.

[DLG02] Thomas Duquesne and Jean-François Le Gall. Random trees, Lévy processes and spatial branching processes. Astérisque, 281:vi+147, 2002.

[DS11] Bertrand Duplantier and Scott Sheffield. Liouville quantum gravity and KPZ. Invent. Math., 185(2):333-393, 2011.

[GGN13] Ori Gurel-Gurevich and Asaf Nachmias. Recurrence of planar graph limits. Ann. of Math. (2), 177(2):761-781, 2013.

[Gro99] Misha Gromov. Metric structures for Riemannian and non-Riemannian spaces, volume 152 of Progress in Mathematics. Birkhäuser Boston Inc., Boston, MA, 1999. Based on the 1981 French original [MR0682063 (85e:53051)], With appendices by M. Katz, P. Pansu and S. Semmes, Translated from the French by Sean Michael Bates.

[GW99] Zhicheng Gao and Nicholas C. Wormald. The size of the largest components in random planar maps. SIAM J. Discrete Math., 12(2):217-228 (electronic), 1999.

[JS12] Svante Janson and Sigurdur Orn Stefansson. Scaling limits of random planar maps with a unique large face. Preprint, arXiv: 1212. 5072, 2012.

[Kai76] William D. Kaigh. An invariance principle for random walk conditioned by a late return to zero. Ann. Probability, 4(1):115-121, 1976.

[Kri05] Maxim Krikun. Local structure of random quadrangulations. Preprint, arXiv: $0512304,2005$. 
[LG99] Jean-François Le Gall. Spatial branching processes, random snakes and partial differential equations. Lectures in Mathematics ETH Zürich. Birkhäuser Verlag, Basel, 1999.

[LG05] Jean-François Le Gall. Random trees and applications. Probab. Surv., 2:245-311 (electronic), 2005.

[LG06] Jean-François Le Gall. A conditional limit theorem for tree-indexed random walk. Stocastic Process. Appl., 116:539-567, 2006.

[LG07] Jean-François Le Gall. The topological structure of scaling limits of large planar maps. Invent. Math., 169(3):621-670, 2007.

[LG10] Jean-François Le Gall. Geodesics in large planar maps and in the Brownian map. Acta Math., 205(2):287-360, 2010.

[LG13] Jean-François Le Gall. Uniqueness and universality of the Brownian map. Ann. Probab., 41(4):2880-2960, 2013.

[LGLJ98] Jean-François Le Gall and Yves Le Jan. Branching processes in Lévy processes: the exploration process. Ann. Probab., 26(1):213-252, 1998.

[LGM11] Jean-François Le Gall and Grégory Miermont. Scaling limits of random planar maps with large faces. Ann. Probab., 39(1):1-69, 2011.

[LGM12] Jean-François Le Gall and Grégory Miermont. Scaling limits of random trees and planar maps. In Probability and statistical physics in two and more dimensions, volume 15 of Clay Math. Proc., pages 155-211. Amer. Math. Soc., Providence, RI, 2012.

[LGP08] Jean-François Le Gall and Frédéric Paulin. Scaling limits of bipartite planar maps are homeomorphic to the 2-sphere. Geom. Funct. Anal., 18(3):893-918, 2008.

[Mie08] Grégory Miermont. On the sphericity of scaling limits of random planar quadrangulations. Electron. Commun. Probab., 13:248-257, 2008.

[Mie13] Grégory Miermont. The Brownian map is the scaling limit of uniform random plane quadrangulations. Acta Math., 210(2):319-401, 2013.

[MM06] Jean-François Marckert and Abdelkader Mokkadem. Limit of normalized quadrangulations: the Brownian map. Ann. Probab., 34(6):2144-2202, 2006.

[MN14] Laurent MÃlnard and Pierre Nolin. Percolation on uniform infinite planar maps. Electron. J. Probab., 19:no. 79, 1-27, 2014.

[PS06] Dominique Poulalhon and Gilles Schaeffer. Optimal coding and sampling of triangulations. Algorithmica, 46(3-4):505527, 2006.

[Sch98] Gilles Schaeffer. Conjugaison d'arbres et cartes combinatoires aléatoires. PhD thesis, Université de Bordeaux 1, 1998. 